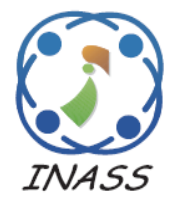

\title{
Hyperchaos Synchronization Using T-S Fuzzy Model Based Synergetic Control Theory
}

\author{
Khalissa Behih $^{1 *} \quad$ Salah Eddine Saadi $^{2} \quad$ Ziyad Bouchama $^{2,3}$ \\ ${ }^{1}$ LSI Laboratory, Department of Electrical Engineering, Ferhat Abbas University of Sétifl, Sétif, Algeria \\ ${ }^{2}$ Department of Electromechanical Engineering, Mohammed El Bachir El-IbrahimiUniversity of Bordj Bou \\ Arréridj, Algeria \\ ${ }^{3}$ QUERE Laboratory, Department of Electrical Engineering, Ferhat Abbas University of Sétif1, Sétif, Algeria \\ * Corresponding author's Email: khalissabehih@univ-setif.dz
}

\begin{abstract}
This paper addresses the design of synergetic control (SC) for synchronizing two chaos systems represented by Takagi-Sugeno (T-S) fuzzy models. Unlike many works published, the present paper provides a new synchronization algorithm of two different hyperchaotic systems which corroborate the given analytical stability proof of the closed-loop systems. The main idea is to represent the chaos systems as an aggregated fuzzy model which compromises a set of linear models in the first place. Afterwards, the synergistic controller is applied to synchronize a nonlinear hyperchaotic (slave) system with another hyperchaotic (master) system. The proposed approach achieves hyperchaos synchronization such that it has a simple structure leading to an easy implementation. Then, theoretical analysis and numerical simulation results are compared to those obtained with a conventional sliding mode (SMC) approach clearly showing the effectiveness of the proposed strategy.
\end{abstract}

Keywords: Chaos, Synchronization, Hyperchaos systems, T-S fuzzy modelling, Synergetic control, Fuzzy synergetic controller.

\section{Introduction}

Chaos control and chaos analysis as well as chaotification in dynamical systems have received increasing attention from research communities and have been studied extensively. Recently, chaotic systems with higher dimensional attractors called hyperchaotic systems have a broad spectrum of applications in the fields of chemical, electrical engineering, computer and information processing [1-5], etc. Moreover, hyperchaotic synchronization has been the subject of strong and fervent attention from the above fields.

Hyperchaotic synchronization is based on the concept of closeness of the frequencies between two hyperchaotic systems that one is a master or drive system and the other one is a slave or response system. This process is realized by the development of adequate control laws ensuring asymptotically zero error between master and slave systems states.
Since the discovery of hyperchaotic synchronization, various control techniques and methods have been proposed, such as passive [6], nonlinear feedback [7], adaptive [8], backstepping [9] and sliding mode [10]. In most of these approaches were not used to synchronize two different hyperchaotic systems due to their different structures and parameter mismatched. However, a fuzzy modeling approach based on synergetic control theory has been developed for hyperchaotic synchronization to overcome the above problem.

Recently, fuzzy control techniques have been widely and successfully used in modeling and control of nonlinear systems [11-13]. Especially, Takagi-Sugeno (T-S) fuzzy model [13-17] has been a popular choice in industrial processes due to its ability to represent the nonlinear system without complex mathematical equations.

The T-S fuzzy model provides a suitable framework for modeling by decomposition of a 
nonlinear system into a collection of local linear models. The individual consequents are linear systems which can be analyzed by using the standard tools of linear systems theory. Indeed, the design of robust control methodologies which use fuzzy system techniques is being steadily more researched and extended, such as the integration of fuzzy sets and synergetic control (SC) can produce enhanced performances $[11,18,19]$ resulting from the combination of the advantages of both techniques. The SC is a most propitious approach based on the invariance feature found in sliding mode control (SMC), yet devoid of its short coming: inherent chattering. Its robustness and its ease in implementation have put forth this fairly new control approach.

In this paper, T-S fuzzy approach is used to present the desired dynamic characteristics of two different hyperchaotic systems. Then, the design of a fuzzy synergetic controller to synchronize the nonlinear systems obtained through the T-S fuzzy technique is undertaken and stability conditions are given. The performance of the newly designed controller is evaluated on the synchronization of hyperchaotic Lu and hyperchaotic Lorenz systems in comparison with the sliding mode control approach.

\section{T-S fuzzy modeling of hyperchaotic systems}

The T-S fuzzy modeling consists of determining validity regions and the corresponding local affine models, so that the overall nonlinear behavior of the system can be appropriately described by fuzzy merging of these models. Fuzzy dynamic model is described by fuzzy if-then rules and will be employed here to deal with the control design problem for the hyperchaotic system. In this section, an application example based on hyperchaotic $\mathrm{Lu}$ system is given to illustrate the effectiveness and advantages of the T-S fuzzy modeling method.

Now, Consider the hyperchaotic Lu system [20, 21] of the form:

$$
\left\{\begin{array}{l}
\dot{y}_{1}=a\left(y_{2}-y_{1}\right)+y_{4} \\
\dot{y}_{2}=-y_{1} y_{3}+c y_{2} \\
\dot{y}_{3}=y_{1} y_{2}+b y_{3} \\
\dot{y}_{4}=y_{1} y_{3}+r y_{4}
\end{array}\right.
$$

Where $y_{1}, y_{2}, y_{3}$ and $y_{4}$ are the state variables and $a, b, c$ and $r$ are real system parameters.

The system Eq. (1) exhibits hyperchaotic behavior $[20,21]$ when the parameter values are: $a=36, b=3, c=20$ and $-0,35 \leq r \leq 1,3$. Assume that $y_{1}(t) \in[-\beta \beta]$ and $\beta>0$, then, the dynamic fuzzy model suitable to the system Eq. (1) can be described as follows:

$$
\begin{aligned}
& \text { Rule1:If } y_{1}(t) \text { is } \xi_{1} \text {, Then } \dot{y}(t)=A_{1} y(t) \\
& \text { Rule2:If } y_{1}(t) \text { is } \xi_{2} \text {, Then } \dot{y}(t)=A_{2} y(t)
\end{aligned}
$$

Where $\xi_{i}(i=1,2)$ are the fuzzy sets and $y(t)=A_{i}$ $y(t)$ is the output from the ith If-Then rules. So, the final output of the fuzzy model of the hyperchaotic system Eq. (1) is inferred as follows:

$$
\dot{y}(t)=\sum_{i=1}^{2} \xi_{i}\left(y_{1}\right) A_{i} y(t)
$$

Where:

$$
A_{1}=\left[\begin{array}{cccc}
-a & a & 0 & 1 \\
0 & c & -\beta & 0 \\
0 & \beta & b & 0 \\
0 & 0 & \beta & r
\end{array}\right], \xi_{1}=0.5+\frac{y_{1}(t)}{2 \beta}
$$

and

$$
A_{2}=\left[\begin{array}{cccc}
-a & a & 0 & 1 \\
0 & c & \beta & 0 \\
0 & -\beta & b & 0 \\
0 & 0 & -\beta & r
\end{array}\right], \xi_{2}=0.5-\frac{y_{1}(t)}{2 \beta}
$$

The following numerical simulations are carried to verify that fuzzy system Eq. (3) indeed has a hyperchaotic behavior.

System responses depicted in Fig. 1 are identical to results obtained and discussed in [20-21]. So, for any region of interest, hyperchaotic Lu system Eq. (1) can be modeled exactly by the fuzzy system Eq. (3).

\section{T-S fuzzy synergetic synchronization of hyperchaotic systems}

Synergetic control (SC) theory offers a control framework based on a theory conceived to control non-linear dynamical processes. The SC techniques $[11,18,19,22]$ can provide a kind of continuous control law, for driving the system states to regions/sets of attraction that correspond to the control purposes, and 60 then force the trajectories to stay on those regions. Some of basic SC concepts will be reviewed here before the proposed T-S fuzzy synergetic control scheme is introduced. First, consider the linear controllable system.

$$
\dot{x}(t)=A x(t)+B u(t)
$$




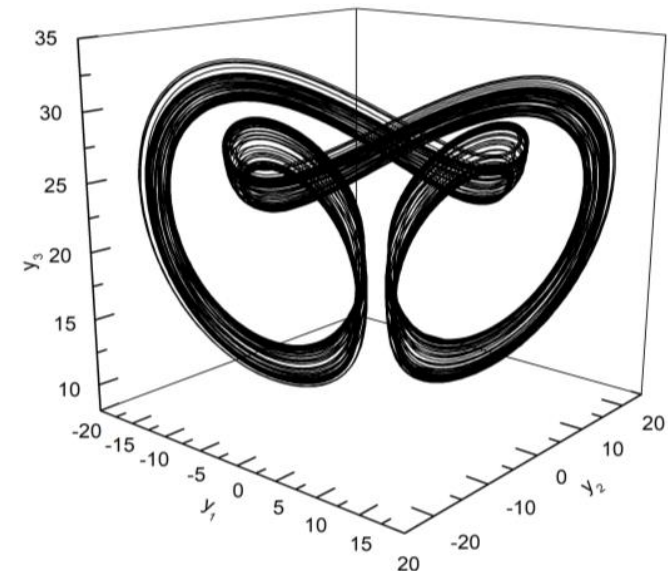

(a)

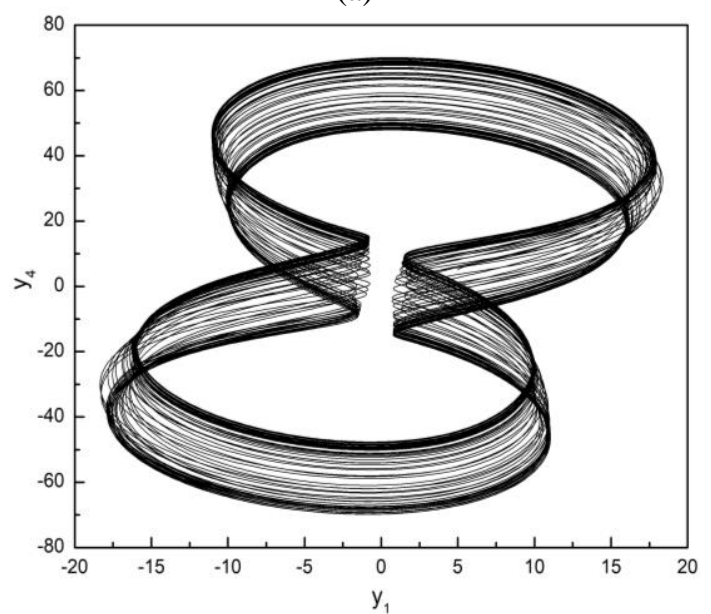

(c)

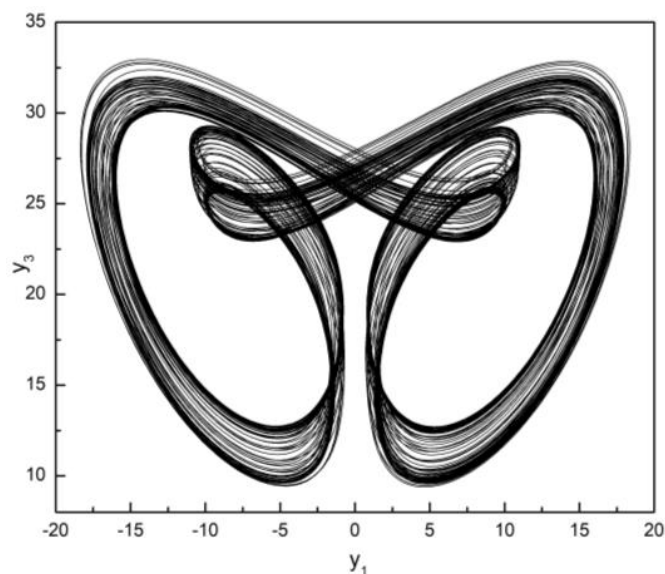

(b)

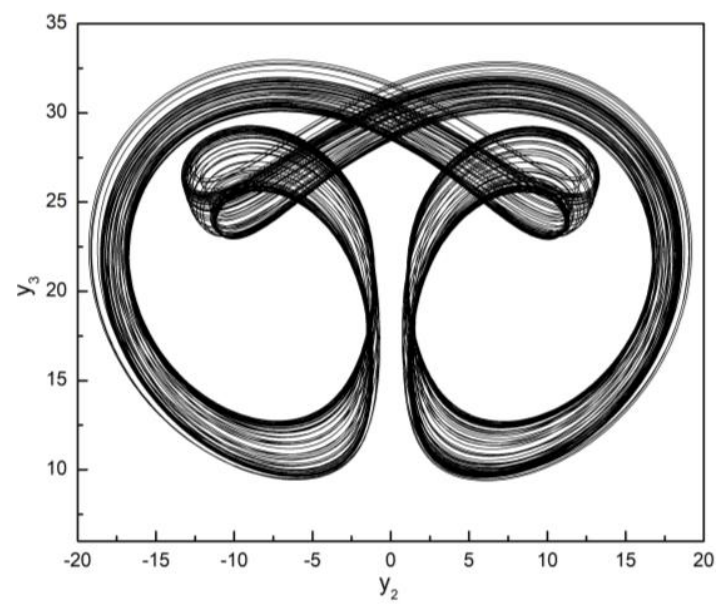

(d)

Figure. 1 Typical dynamic behaviors of fuzzy system (3). Hyperchaotic attractors of the system: (a) in the space $y_{1}, y_{2}, y_{3}$, (b) in the plane $y_{1}, y_{3},(\mathrm{c})$ in the plane $y_{1}, y_{4}$, and (d) in the plane $y_{2}, y_{3}$

Where $x \epsilon \mathfrak{R}^{n x l}$ is system state, $A \epsilon \mathfrak{R}^{n x n}$ and $B \epsilon$ $\mathfrak{R}^{n x r}$ are constant matrices and $u \in \mathfrak{R}^{n x l}$ is the synergetic control to be designed. Basically, The SC synthesis begins by defining a designer chosen macro-variable that need to be zero. The macrovariable is function of system variables which can be defined as:

$$
\sigma(x)=\kappa\left(x-y_{d}\right)
$$

Where $y_{d}=\left[\begin{array}{lllll}y_{1 d} & y_{2 d} & \ldots & y_{n d}\end{array}\right] \in \mathfrak{R}^{\mathrm{nx} 1}$ is the desired output signal and is a constant matrix to be determined.

Hence, to attain the control purposes, attractor is introduced where the macro-variable Eq. (5) is equal to zero. The SC gives an equation which can be used for creating dynamical system with attractor at $\sigma=0$ which can be stated as:

$$
\dot{\sigma}+\tau \sigma=0 \quad \tau>0
$$

Using Eq. (5) to solve the system Eq. (4) with the evolution condition Eq. (6), then one can have:

$$
\kappa A x(t)+\kappa B u(t)=-\tau \sigma+\kappa \dot{y}_{d}
$$

The resulting synergetic control law can be expressed as:

$$
u(t)=-(\kappa B)^{-1}\left[\kappa A x(t)+\tau \sigma-\kappa \dot{y}_{d}\right], \quad(\kappa B) \neq 0
$$

Next, synergetic controller design procedure for the synchronization of hyperchaotic systems is presented. Choose controlled $\mathrm{Lu}$ hyperchaotic system as drive system and considered Lorenz hyperchaotic system as a response system which has the following fuzzy model [23].

$$
\dot{x}(t)=\sum_{i=1}^{2} \xi_{i}^{\prime}\left(x_{1}\right) A_{i}^{\prime} x(t)
$$

The following structure of fuzzy rules is used to provide the synergetic control law which synchronize the two hyperchaotic systems. 
Rulel:if $y_{1}(t)$ is $\xi_{1}$ and $x_{1}(t)$ is $\xi_{1}^{\prime}$,

$$
\begin{gathered}
\text { Then } \dot{y}(t)=A_{1} y(t)+B_{1} u_{l}(t) \text { and } \\
u_{1}(t)=-\left(\kappa B_{1}\right)^{-1}\left[\kappa A_{1} y(t)+\tau_{1} \sigma-\kappa A_{1}^{\prime} x(t)\right]
\end{gathered}
$$

Rule2:if $y_{1}(t)$ is $\xi_{2}$ and $x_{1}(t)$ is $\xi_{2}^{\prime}$,

$$
\begin{gathered}
\text { Then } \dot{y}(t)=A_{2} y(t)+B_{2} u_{2}(t) \text { and } \\
u_{2}(t)=-\left(\kappa B_{2}\right)^{-1}\left[\kappa A_{2} y(t)+\tau_{2} \sigma-\kappa A_{2}^{\prime} x(t)\right]
\end{gathered}
$$

Thus, the general fuzzy model and the overall controller can be represented by

$$
\begin{gathered}
\dot{y}(t)=\sum_{i=1}^{2} \xi_{i}\left(y_{1}\right) A_{i} y(t)+\sum_{i=1}^{2} \xi_{i}\left(y_{1}\right) B_{i} u(t) \\
u(t)=\sum_{\mathrm{i}=1}^{2} \xi_{i}\left(y_{i}\right) u_{i}(t)-\kappa \sum_{\mathrm{i}=1}^{2} F_{i}\left(x_{i}\right) A_{i}^{\prime} x(t)
\end{gathered}
$$

Where:

$$
u_{i}(t)=-\left(\kappa B_{i}\right)^{-1}\left[\kappa A_{i} y(t)+\tau_{i} \sigma\right]
$$

By using the Lyapunov stability analysis, the following theorems for the stabilization and the synchronization of the hyperchaotic systems are verified.

\subsection{Theorem 1}

Consider the synchronization problem of the hyperchaotic Lorenz system [23] and the hyperchaotic Lu system (1), if the fuzzy SC action (13) is used and $\kappa B_{i}=\kappa B_{j}=\kappa B$ for $i \neq j$, then the drive and response systems are asymptotically synchronized.

\subsection{Proof}

Let the Lyapunov function candidate be $Z=0,5 \sigma^{\mathrm{T}} \sigma$. Therefore,

$$
\dot{Z}=\sigma^{T} \dot{\sigma}=\sigma^{T} \kappa(\dot{y}-\dot{x})
$$

Substituting Eq. (11) into Eq. (14) yields

$$
\begin{aligned}
\dot{Z}=\sigma^{T} \kappa & \left(\sum_{i=1}^{2} \xi_{i}\left(y_{1}\right) A_{i} y(t)\right. \\
& \left.+\kappa \sum_{i=1}^{2} \xi_{i}\left(y_{1}\right) B_{i} u(t)-\dot{x}\right)
\end{aligned}
$$

Then, using Eq. (12) and substituting the conditions of the theorem 1 into Eq. (15) yields

$$
\begin{gathered}
\dot{Z}=\sigma^{T} \kappa\left(\sum_{i=1}^{2} \xi_{i}\left(y_{1}\right) A_{i} y(t)+\kappa B \sum_{i=1}^{2} \xi_{i}\left(y_{1}\right)\right. \\
\leq-2 \sum_{i=1}^{2} \xi_{i}\left(y_{1}\right) \tau_{i} Z(t) \leq 0
\end{gathered}
$$

Since for $(\kappa B)^{-1} \neq 0, \xi_{i}\left(y_{1}\right)>0$ and $\tau_{i}>0$, then (16) indicates that the stats $x_{i}$ of the response system and the stats $y_{i}$ of drive system are synchronized asymptotically.

\section{Robustness analysis}

Next, the robustness of the proposed fuzzy synergetic control strategy is improved when the above theorem conditions $\kappa B_{i}=\kappa B_{j}=\kappa B$ for for $i \neq j$ are not easily applicable.

\subsection{Theorem 2}

Consider the synchronization problem of the hyperchaotic Lorenz system [23] and the hyperchaotic Lu system Eq. (1), if the fuzzy SC action Eq. (13) is redesigned as:

$$
\begin{aligned}
& u_{i}(t)=u^{k}(t) \\
&=-\left(\kappa B_{k}\right)^{-1}\left[\kappa A_{k} y(t)+\tau_{k} \sigma-\kappa \dot{x}\right] \\
& \mathrm{k}=\left\{i: \max \left[\xi_{1}, \ldots, \xi_{i}, \ldots, \xi_{r}\right]\right\}
\end{aligned}
$$

Where $u^{k}(t)$ is the dominant control law in the fuzzy rules Eq. (10) and the control $\tau_{k}$ satisfying:

$$
\tau_{k}>\tau_{i}
$$

Where :

$$
\tau_{i}=\frac{\left\|\kappa A_{i}-\kappa B_{i}\left(\kappa B_{k}\right)^{-1} \kappa A_{k}\right\|}{\|\kappa\| Q_{\text {min }}\left(\kappa B_{i}\left(\kappa B_{k}\right)^{-1}+\left(\kappa B_{i}\left(\kappa B_{k}\right)^{-1}\right)^{T}\right)}>0
$$

Then the two hyperchaotic systems are synchronized asymptotically.

\subsection{Proof}

Let's consider Lyapunov function candidate: $Z=0.5 \sigma^{\mathrm{T}} \sigma$, whose time derivative leads to:

$$
\dot{Z}=\sigma^{\mathrm{T}} \dot{\sigma}
$$

Then,

$$
\begin{array}{r}
\dot{Z}=\sigma^{T} \kappa\left(\sum_{i=1}^{2} \xi_{i}\left(y_{1}\right) A_{i} y(t)+\sum_{i=1}^{2} \xi_{i}\left(y_{1}\right) B_{i} u(t)\right. \\
\left.-\sum_{i=1}^{2} \xi_{i}^{\prime}\left(x_{i}\right) A_{i}^{\prime} x(t)\right) \\
=\sigma^{T}\left(\sum_{i=1}^{2} \xi_{i}\left(y_{1}\right) A_{i} y(t)-\sum_{i=1}^{2} \xi_{i}\left(y_{1}\right) \kappa B_{i}\right. \\
\left.\left(\left(\kappa B_{k}\right)^{-1}\left[\kappa A_{k} y(t)+\tau_{k} \sigma\right]\right)\right) \\
=\sum_{i=1}^{2} \xi_{i}\left(y_{1}\right) \sigma^{T}\left(\left(\kappa A_{i}-\kappa B_{i}\left(\kappa B_{k}\right)^{-1} \kappa A_{k}\right) y(t)\right. \\
\left.-\kappa B_{i}\left(\kappa B_{k}\right)^{-1} \tau_{k} \sigma\right)
\end{array}
$$


Let $Q_{\min }>0$ denote the smallest eigenvalue of the matrix $\left(\left(\kappa B_{i}\left(\kappa B_{k}\right)^{-1}\right)^{\mathrm{T}}+\left(\kappa B_{i}\left(\kappa B_{k}\right)^{-1}\right)\right.$. Then, the minimization of $\dot{Z}$ can be obtained as:

$$
\begin{aligned}
& \dot{Z} \leq-\sum_{i=1}^{2} \xi_{i}\left(y_{1}\right)\|y(t)\|\left(Q_{m i n}\left(\kappa B_{i}\left(\kappa B_{k}\right)^{-1}\right)^{T}\right. \\
& \left.\quad+\kappa B_{i}\left(\kappa B_{k}\right)^{-1} \tau_{k}\|\kappa\|-\left\|\kappa A_{i-} \kappa B_{i}\left(\kappa B_{k}\right)^{-1} \kappa A_{k}\right\|\right)\|\sigma\| \\
& \quad \leq 0
\end{aligned}
$$

Where the gain condition Eq. (18) has been applied. Since $\dot{Z} \leq 0$ it can be concluded that the asymptotical synchronization and the global stability are guaranteed.

\section{Type-style and fonts simulation result}

In this section, an application example based on the hyperchaotic Lorenz system is given to illustrate the effectiveness and advantages of the proposed method. The simplified schematic diagram of the proposed synchronization method and the interconnection of the techniques used are illustrated in Fig. 2.

The fuzzy model of hyperchaotic Lu system is obtained by linearizing the nonlinear Eq. (1) over two operation points as given in section 2. In this paper, the hyperchaotic Lorenz system has the following form [23]:

$$
\left\{\begin{array}{l}
\dot{x}_{1}=a^{\prime}\left(x_{2}-x_{1}\right) \\
\dot{x}_{2}=b^{\prime} x_{1}-x_{2}-x_{1} x_{3}+x_{4} \\
\dot{x}_{3}=c^{\prime} x_{1}+x_{1} x_{2} \\
\dot{x}_{4}=k_{1} x_{1}+k_{2} x_{2}
\end{array}\right.
$$

Where $x_{1}, x_{2}, x_{3}$ and $x_{4}$ are the state variables and $a^{\prime}, b^{\prime}, c^{\prime}, k_{1}$ and $k_{2}$ are constant parameters. The system Eq. (22) exhibits hyperchaotic behavior when the parameter values are [23]: $a^{\prime}=10, b^{\prime}=28$, $c^{\prime}=8 / 3, k_{1}=-9.3$ and $k_{2}=1$.

The T-S fuzzy model for the hyperchaotic Lorenz system Eq. (22) is introduced in [24]. Where:

$$
A_{1}^{\prime}=\left[\begin{array}{cccc}
-a^{\prime} & a^{\prime} & 0 & 0 \\
b^{\prime} & -1 & -\alpha & 1 \\
-c^{\prime} & \alpha & 0 & 0 \\
k_{1} & k_{2} & 0 & 0
\end{array}\right], \xi_{1}^{\prime}=\frac{1}{2}\left(1+\frac{x_{1}(t)}{2 \alpha}\right)
$$

And

$$
A_{2}^{\prime}=\left[\begin{array}{cccc}
-a^{\prime} & a^{\prime} & 0 & 0 \\
b^{\prime} & -1 & \alpha & 1 \\
-c^{\prime} & -\alpha & 0 & 0 \\
k_{1} & k_{2} & 0 & 0
\end{array}\right], \xi_{2}^{\prime}=\frac{1}{2}\left(1-\frac{x_{1}(t)}{2 \alpha}\right)
$$



Figure. 2 Overall scheme of the fuzzy synergetic controller for synchronization of hyperchaotic systems

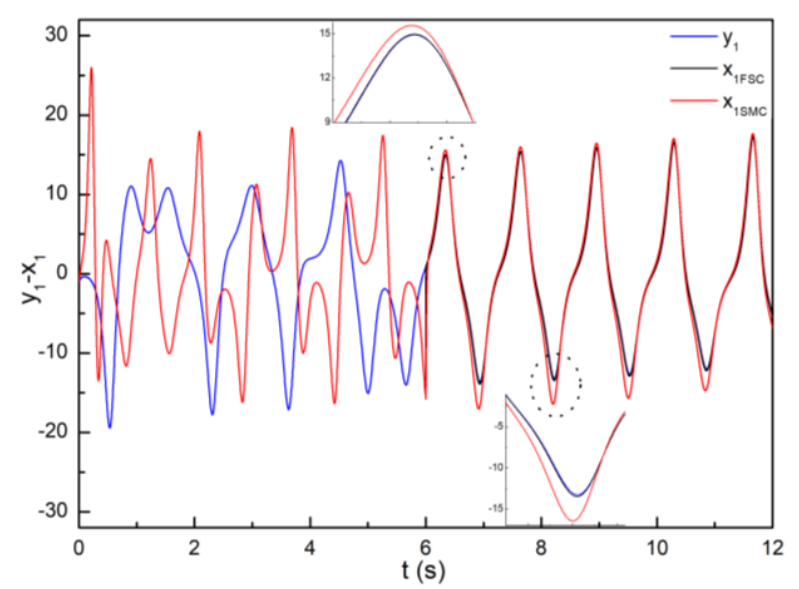

Figure. 3 Trajectories of the drive system $y_{1}$ and the response system $x_{1}$

To obtain an effective fuzzy synergetic synchronization between the above hyper-chaotic systems; the control gain is chosen to be $\tau_{k}=9$ which is greater than all. Also $Q_{\min }>0$ is satisfied for all $i$ and $k$.

Firstly, hyperchaotic Lu system is regarded as a drive system, the initial conditions of the drive and response systems are selected as $x(0)=y(0)=\left[\begin{array}{ll}-1 & 1\end{array}-\right.$ $0.10 .1]$.

The simulation results of the proposed synchronization algorithm are provided in comparison with the results of the SMC method [25] and time trajectories of the synchronization state systems are shown in Fig. 6 to 9. The controller is off for $t<6 s$ and is on for $t \geq 6 s$.

Obviously, the dynamics of synchronization variable states between hyperchaotic Lu system $\left(y_{i}\right.$, 


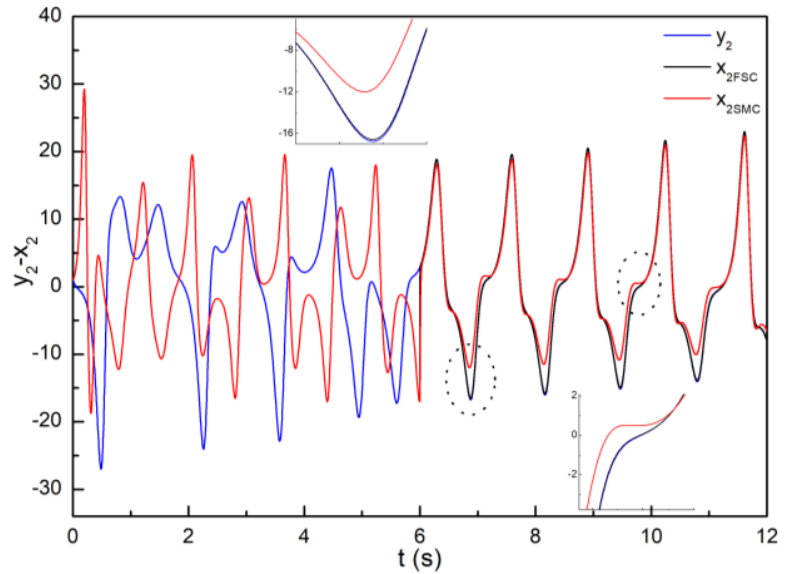

Figure. 4 Trajectories of the drive system $y_{2}$ and the response system $x_{2}$

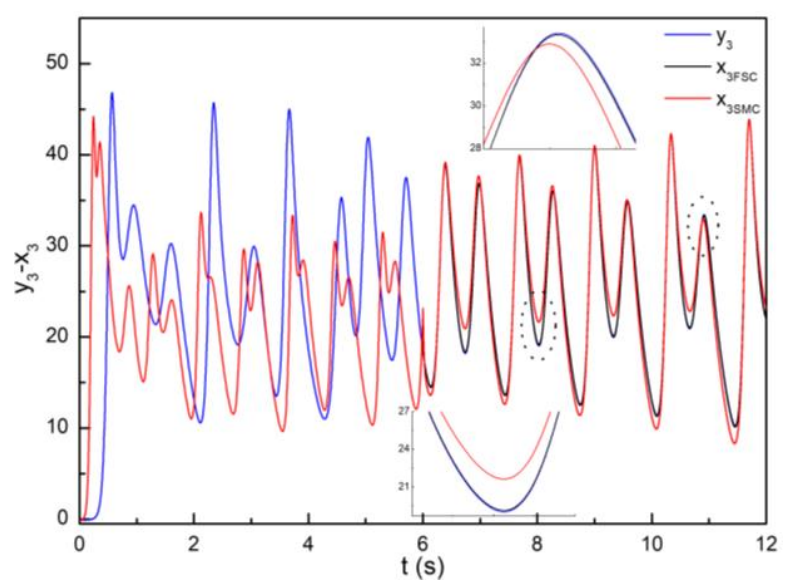

Figure. 5 Trajectories of the drive system $y_{3}$ and the response system $x_{3}$

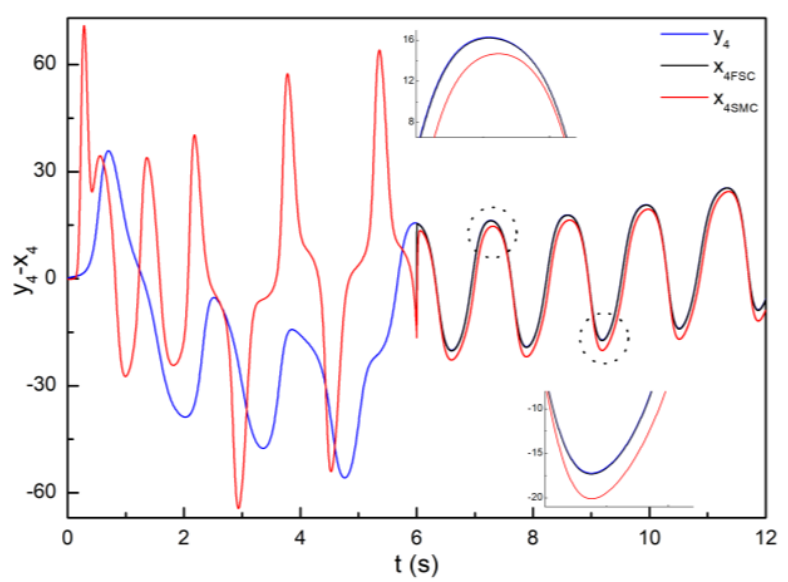

Figure. 6 Trajectories of the drive system $y_{4}$ and the response system $x_{4}$

drive system) and hyperchaotic Lorenz system $\left(x_{i}\right.$, response system) are indeed achieved with chaos synchronization. It is obtained from zoomed time windows in Fig. 6 to 9 that the FSC method provides a faster and more accurate convergence to the master states compared to the other method.

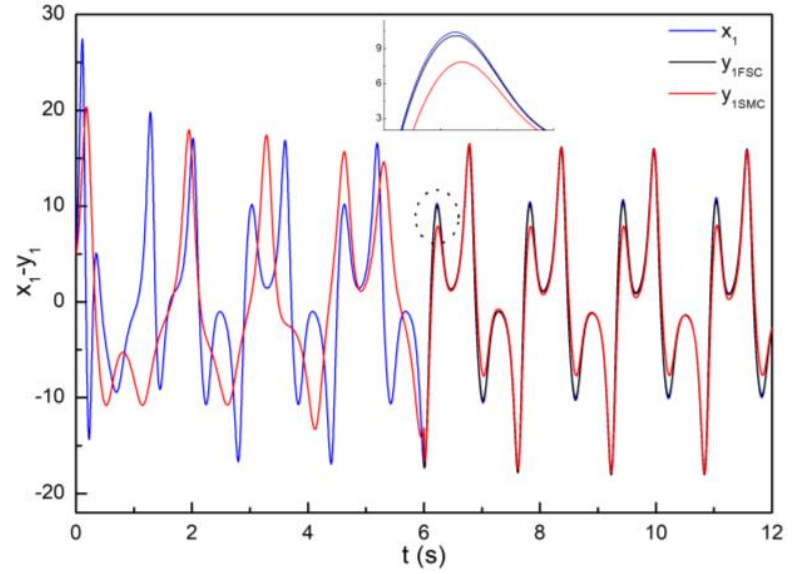

Figure. 7 Trajectories of the drive system $x_{1}$ and the response system $y_{l}$

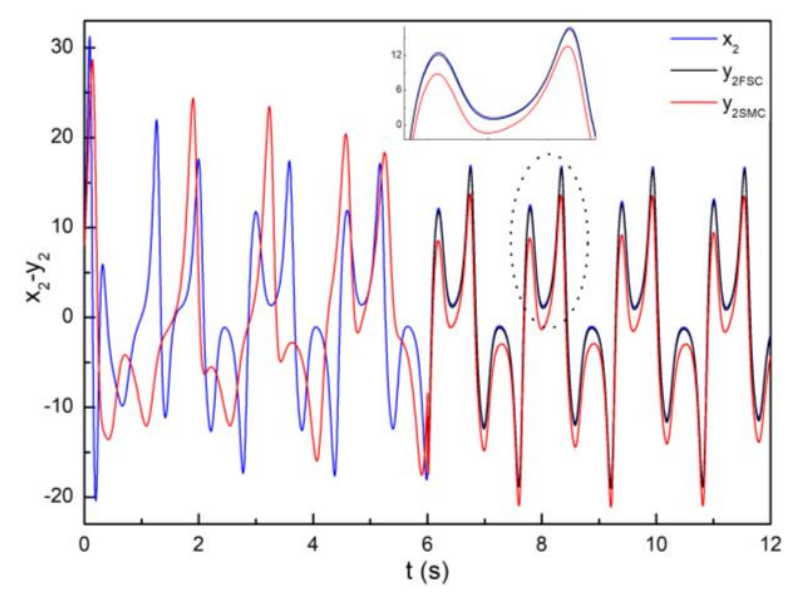

Figure. 8 Trajectories of the drive system $x_{2}$ and the response system $y_{2}$



Figure. 9 Trajectories of the drive system $x_{3}$ and the response system $y_{3}$

Secondly, hyperchaotic Lorenz system is regarded as a drive system, the initial conditions of the drive and response systems are selected as $x(0)=y(0)=\left[\begin{array}{llll}5 & 8 & -1 & -2\end{array}\right]$.

Numerical simulation results are shown in Fig. 10 to 13 , which shows that the proposed method is successful in synchronizing the two systems compared with the results of the SMC method. 


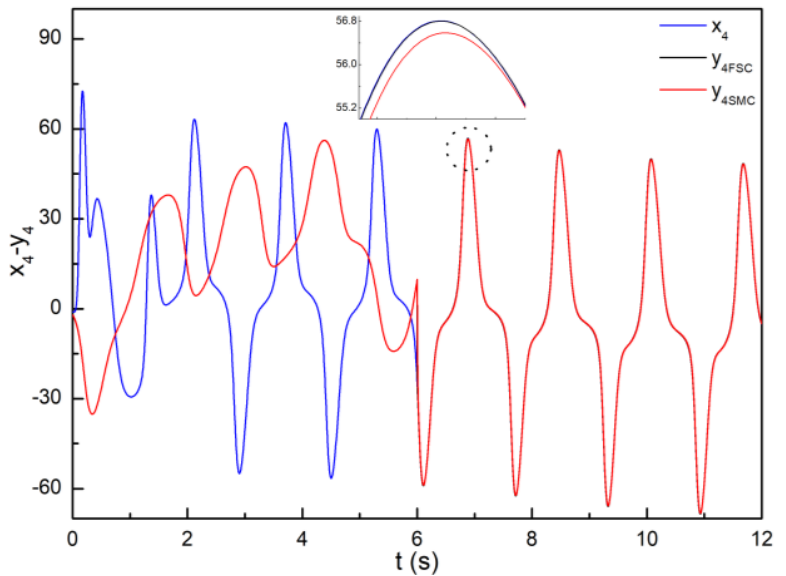

Figure. 10 Trajectories of the drive system $x_{4}$ and the response system $y_{4}$

\section{Conclusion}

The objective for this investigation was to propose a robust controller for synchronization of hyperchaotic systems based on synergetic control theory combined with Takagi-Sugeno fuzzy model properties, which is carried out through numerical simulations. Furthermore, a Lyapunov based synthesis method was developed to ensure the asymptotic synchronization and stability of the general fuzzy models. Hyperchaotic $\mathrm{Lu}$ and hyperchaotic Lorenz systems was used to illustrate the performance of the proposed control strategy. Simulation results show the effectiveness and the robustness of the proposed approach in chaos synchronization problem comparatively to other considered controllers. For future work, the proposed scheme should be used for an efficient synchronization or anti-synchronization of more types of hyperchaotic systems.

\section{Conflicts of Interest}

The authors declare no conflict of interest.

\section{Author Contributions}

The paper conceptualization, methodology, software, validation, formal analysis, investigation, resources, data curation, writing - original draft preparation, writing-review and editing, visualization, have been done by all authors. The supervision, and project administration, have been done by 1 st author.

\section{References}

[1] F. Yang, J. Mou, Y. Cao, and R. Chu, "An Image Encryption Algorithm Based on BP Neural Network and Hyperchaotic System",
China Communications, Vol. 17, No. 5, pp. 2128, 2020.

[2] D. Zhu, R. Wang, C. Liu, and J. Duan, "Synchronization of Chaotic-Oscillation Permanent Magnet Synchronous Generators Networks via Adaptive Impulsive Control", IEEE Transactions on Circuits and Systems II: Express Briefs, Vol. 67, No. 10, pp. 2194-2198, 2019.

[3] H. Acikgoz and R. Mittra, "Stochastic Polynomial Chaos Expansion Analysis of a Split-Ring Resonator at Terahertz Frequencies", IEEE Transactions on Antennas and Propagation, Vol. 66, No. 4, pp. 2131-2134, 2018.

[4] S. Madbushi, R. Raut, and M. S. S. Rukmini, "A Novel Chaotic Communication Based Test Signal Approach for Identification of Primary User Emulation Attack in Cognitive Radio Networks", International Journal of Intelligent Engineering and Systems, Vol. 11, No. 2, pp. 57-67, 2018.

[5] S. Koppu and M. Viswanatham, "2D Chaotic Map Based on 2D Adaptive Grey Wolf Algorithm for Ultra Sound Medical Image Security", International Journal of Intelligent Engineering and Systems, Vol. 10, No. 1, pp. 104-113, 2017.

[6] A. L. Fradkov and A. Y. Markov, "Adaptive Synchronization of Chaotic Systems Based on Speed Gradient Method and Passification", IEEE Transactions on Circuits and Systems I: Fundamental Theory and Applications, Vol. 44, No. 10, pp. 905-912, 1997.

[7] J. H. Pérez, M. Figueroa, S. A. Rodríguez, and A. López, "Synchronization of Chaotic Akgul System by Means of Feedback Linearization and Pole Placement", IEEE Latin America Transactions, Vol. 15, No. 2, pp. 249-256, 2017.

[8] J. Lu, R. Wei, X. Wang, and Z. Wang, "Backstepping Control of Discrete-Time Chaotic Systems with Application to The Henon System", IEEE Transactions on Circuits and Systems I: Fundamental Theory and Applications, Vol. 48, No. 11, pp. 1359-1363, 2001.

[9] K. Khettab, Y. Bensafia, and S. Ladaci, "Chattering Elimination in Fuzzy Sliding Mode Control of Fractional Chaotic Systems Using a Fractional Adaptive Proportional Integral Controller", International Journal of Intelligent Engineering and Systems, Vol. 10, No. 5, pp. 255-266, 2017. 
[10] X. Meng, Z. Wu, C. Gao, B. Jiang, and H. R. Karimi, "Finite-time Projective Synchronization Control of Variable-Order Fractional Chaotic Systems via Sliding Mode Approach", IEEE Transactions on Circuits and Systems II: Express Briefs, Vol. 68, No. 7, pp. 2503-2507, 2021.

[11] Z. Bouchama, A. Khatir, S. Benaggoune, and M. N. Harmas, "Design and Experimental Validation of An Intelligent Controller for DcDc Buck Converters", Journal of the Franklin Institute, Vol. 357, No. 15, pp. 10353-10366, 2020.

[12] H. N. Abdullah, "An Improvement in LQR Controller Design based on Modified Chaotic Particle Swarm Optimization and Model Order Reduction", International Journal of Intelligent Engineering \& Systems, Vol. 14, No. 1, pp. 157-168, 2021.

[13] H. L. Lyu, W. Wang, X. P. Liu, and Z. Q. Wang, "Modeling of Multivariable Fuzzy Systems By Semitensor Product", IEEE Transactions on Fuzzy Systems, Vol. 28, No. 2, pp. 228-235, 2019.

[14] W. Zou, C. Li, and N. Zhang, "AT-S Fuzzy Model Identification Approach Based on a Modified Inter Type-2 FRCM Algorithm", IEEE Transactions on Fuzzy Systems, Vol. 26, No. 3, pp. 1104-1113, 2017.

[15] K. Ouarid, A. E. Assoudi, M. Essabre, and E. E. Yaagoubi, "Fuzzy Observer Design for Fault Estimation for Discrete-time Takagi-Sugeno Singular Models with Unmeasurable Premise Variables", International Journal of Intelligent Engineering and Systems, Vol. 14, No. 4, pp. 514-523, 2021.

[16] X. L. Wang, W. X. Xie, and L. Q. Li, "Interacting Ts Fuzzy Particle Filter Algorithm for Transfer Probability Matrix of Adaptive Online Estimation Model", Digital Signal Processing, Vol. 110, pp. 102944, 2021.

[17] F. Abdelmalki and N. Ouaaline, "The Fuzzy Tracking Control of Output Vector of Double Fed Induction Generator DFIG via T-S Fuzzy Model", International Journal of Intelligent Engineering and Systems, Vol. 11, No. 1, pp. 113-121, 2018.

[18] Z. Bouchama, N. Essounbouli, M. N. Harmas, A. Hamzaoui, and K. Saoudi, "Reaching Phase Free Adaptive Fuzzy Synergetic Power System Stabilizer", International Journal of Electrical Power \& Energy Systems, Vol. 77, pp. 43-49, 2016.

[19] Z. Bouchama and M. N. Harmas, "Optimal Robust Adaptive Fuzzy Synergetic Power
System Stabilizer Design", Electric Power Systems Research, Vol. 83, No. 1, pp. 170-175, 2012.

[20] A. Chen, J. Lu, J. Lü, and S. Yu, "Generating Hyperchaotic Lü Attractor Via State Feedback Control", Physica A: Statistical Mechanics and its Applications, Vol. 364, pp. 103-110, 2006.

[21] M. T. Yassen, "Synchronization Hyperchaos of Hyperchaotic Systems", Chaos, Solitons \& Fractals, Vol. 37, No. 2, pp. 465-475, 2008.

[22] S. Lu, C. Tian, and P. Yan, "Adaptive Extended State Observer-Based Synergetic Control for A Long-Stroke Compliant Microstage with Stress Stiffening", IEEE/ASME Transactions on Mechatronics, Vol. 25, No. 1, pp. 259-270, 2019.

[23] Q. Yang, K. Zhang, and G. Chen, "Hyperchaotic Attractors from A Linearly Controlled Lorenz System", Nonlinear Analysis: Real World Applications, Vol. 10, No. 3, pp. 1601-1617, 2009.

[24] J. Wang, X. Xiong, M. Zhao, and Y. Zhang, "Fuzzy Stability and Synchronization of Hyperchaos Systems", Chaos, Solitons \& Fractals, Vol. 35, No. 5, pp. 922-930, 2008.

[25] J. J. E. Slotine and W. Li, Applied Nonlinear Control, Vol. 199, Englewood Cliffs, NJ: Prentice Hall, New Jersey, 1991. 